\title{
Politique énergétique et facteur 4 : instruments et outils de régulation à disposition des collectivités
}

Camille Chanard, Marie-Hélène de Sède-Marceau et Micael Robert

\section{(2) OpenEdition}

Journals

Édition électronique

URL : http://journals.openedition.org/developpementdurable/8776

DOI : 10.4000/developpementdurable.8776

ISSN : 1772-9971

Éditeur

Association DD\&T

Référence électronique

Camille Chanard, Marie-Hélène de Sède-Marceau et Micael Robert, « Politique énergétique et facteur 4 : instruments et outils de régulation à disposition des collectivités », Développement durable et territoires [En ligne], Vol. 2, n 1 | Mars 2011, mis en ligne le 23 février 2011, consulté le 19 avril 2019 URL : http://journals.openedition.org/developpementdurable/8776 ; DOI : 10.4000/ developpementdurable.8776

Ce document a été généré automatiquement le 19 avril 2019

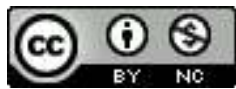

Développement Durable et Territoires est mis à disposition selon les termes de la licence Creative Commons Attribution - Pas d'Utilisation Commerciale 4.0 International. 


\title{
Politique énergétique et facteur 4 : instruments et outils de régulation à disposition des collectivités
}

\author{
Camille Chanard, Marie-Hélène de Sède-Marceau et Micael Robert
}

$1 \quad \mathrm{La}^{1}$ relation entre changement climatique et émissions de gaz à effet de serre a été mise en lumière par le Groupe d'experts intergouvernemental sur l'évolution du climat (GIEC) depuis la fin des années 1980. Les travaux du GIEC ont en particulier établi un rapport entre l'utilisation des énergies fossiles (charbon, pétrole, gaz naturel), qui représentent environ $81 \%$ de la consommation totale d'énergie à l'échelle mondiale (IEA, 2009:6), et l'accroissement du dioxyde de carbone $\left(\mathrm{CO}_{2}\right.$, l'un des principaux gaz à effet de serre avec la vapeur d'eau, le méthane, le protoxyde d'azote et l'ozone) dans l'atmosphère (Watson, $1996: 12$ ).

2 Face à cette réalité, plusieurs engagements internationaux visant une réduction des émissions des gaz à effet de serre (GES) ont été pris : l'Appel de la Haye signé par 43 pays en 1989; la Convention-cadre des Nations Unies sur le changement climatique - issue de la Conférence de la Terre de Rio de Janeiro en 1992 - qui a été ratifiée par 189 pays; le Protocole de Kyoto, signé en 1997, entré en vigueur en 2005 et ratifié à ce jour par 172 pays. Ces objectifs ont ensuite été déclinés au niveau européen puis aux échelons nationaux et ont donné lieu à la rédaction de divers documents d'orientation. En France, le Livre blanc sur les Énergies de novembre 2003 introduit le principe de facteur 4, soit la division par quatre du niveau des émissions de gaz à effet de serre de 1990 à l'horizon 2050. Cet objectif évoqué dans le Plan Climat national de 2004 a été repris dans l'article 2 de la loi de programme fixant les orientations de la politique énergétique du 13 juillet 2005, qui « vise à diminuer de $3 \%$ par an en moyenne les émissions de gaz à effet de serre de la France » (art. 2). Par ailleurs, le Parlement européen a voté en décembre 2008, le « Paquet climat-énergie » qui engage les 27 pays de l'Union à la fois sur l'énergie et sur le climat. Celui-ci fixe les objectifs 20-20-20 à atteindre avant 2020 : une réduction de 20\% des émissions de GES (sur la base des émissions de 1990)², une amélioration de 20\% de l'efficacité énergétique, 
tandis que $20 \%$ de l'approvisionnement énergétiques devra être couvert par des énergies renouvelables.

3 Pour atteindre ces objectifs, il semble indispensable d'intégrer la thématique «énergieclimat» dans la totalité des politiques publiques touchant à la fois aux dimensions économiques, sociales, technologiques ainsi qu'aux politiques d'aménagement des territoires et de protection de l'environnement, et d'impliquer l'ensemble des acteurs, en s'appuyant en particulier sur les échelons locaux. La mise en place de telles politiques transversales et globales va nécessiter un réel changement de culture dans les institutions françaises, en particulier pour les collectivités locales. Les politiques énergétiques ne peuvent plus être seulement techniques, elles doivent concerner l'ensemble des aménagements et actions réalisées à tous les niveaux. Nous nous interrogerons dans cet article sur la façon dont les collectivités locales peuvent intégrer la thématique énergie-climat dans l'ensemble de leurs politiques publiques. Sur quels outils peuvent-elles s'appuyer pour construire des politiques transversales et comment doivent-elles s'organiser?

5 La première partie permettra de démontrer la nécessité d'une implication forte des échelons locaux dans la lutte contre le changement climatique et d'une approche transversale, tant au niveau des politiques publiques que des secteurs d'activité. Elle mettra également en évidence la nécessité d'une articulation efficace entre les différents niveaux de compétence et d'intervention. La deuxième partie sera consacrée aux dispositifs pouvant être mobilisés par les collectivités dans leurs différents domaines de compétences. Après avoir effectué une comparaison des démarches permettant de structurer les politiques énergétiques, nous proposerons un panorama complet des outils à disposition des collectivités en recensant aussi bien les documents obligatoires que les démarches volontaires. Ceci nous permettra de montrer que de nombreux outils existent déjà, bien qu'ils n'aient pas tous été conçus au départ pour la thématique énergie-climat. Enfin, dans une troisième partie, nous nous interrogerons sur les échelles de compétences, sur la compatibilité des différents documents de planification et sur le nécessaire décloisonnement des thématiques et des acteurs.

\section{Pour une approche territoriale de la problématique " énergie-climat »}

\subsection{Aménagement du territoire et consommations énergétiques}

6 Les territoires, selon leurs structures et leurs modes de fonctionnement, sont plus ou moins générateurs de consommations ou d'économies d'énergie, et doivent être organisés et gérés en fonction de leurs caractéristiques et spécificités.

Notons tout d'abord que "l'utilisation de l'énergie hors biomasse représente chaque année en moyenne $69 \%$ des émissions de GES pour la France entière alors qu'en ne considérant que les émissions de $\mathrm{CO}_{2}$, l'utilisation de l'énergie hors biomasse constitue à elle seule entre $93 \%$ et $95 \%$ des émissions de $\mathrm{CO}_{2}$ pour la France entière " (CITEPA, 2010: 63). Si l'on se penche plus particulièrement sur le milieu urbain, le rapport Stern (2006:457) a montré que $78 \%$ des émissions de carbone dues à l'activité humaine provenait des villes. Trois secteurs sont particulièrement concernés et doivent accroître leurs efforts pour atteindre les objectifs énergie-climat internationaux : le bâtiment, les transports et l'aménagement-urbanisme. 
Les deux secteurs des bâtiments et des transports sont à l'origine de plus de la moitié des émissions de GES (CITEPA, $2010: 63$ ), tandis que l'aménagement détermine la répartition des activités sur le territoire et les transports induits, à la fois pour les distances parcourues et pour la part modale, soit l'usage et la maitrise de l'espace. Si l'on ne considère que le $\mathrm{CO}_{2}$, le secteur des transports représentait en 2008 pour la France entière $33 \%$ des émissions (23\% pour le seul transport routier), devant le secteur résidentiel/ tertiaire/commercial/institutionnel, qui comptait pour $25 \%$, l'industrie manufacturière (19\%) et les industries de l'énergie (16\%). La tendance globale est identique pour l'ensemble des GES (CITEPA, $2010: 63$ ).

8 Une diminution des émissions de GES doit donc essentiellement passer par une meilleure efficacité énergétique et une plus grande maîtrise de la consommation d'énergie. L'accent n'est plus seulement mis sur la technique, mais sur le changement de comportement et sur la manière dont nous devons penser l'espace et l'aménagement du territoire ${ }^{3}$. Et si les politiques de transport sont quasiment indissociables des politiques énergétiques (bien qu'on ne se soit pas inquiété de cette problématique lors des périodes d'«énergie abondante »), le secteur du bâtiment en revanche ne s'est guère soucié d'énergie jusqu'il y a peu. L'énergie était jusqu'à présent peu chère et il n'y avait pas de réel enjeu à consommer moins d'énergie pour un service rendu (Jancovici, 2004). L'augmentation du prix de l'énergie, et avec elle l'apparition de la notion de précarité énergétique, ainsi que la prise de conscience du changement climatique, ont placé le secteur du bâtiment comme l'un des enjeux prioritaires dans la réduction des consommations énergétiques et la diminution des émissions des $\mathrm{CO}_{2}$.

9 Ainsi, les collectivités et leurs groupements occupent une place centrale dans la mise en œuvre des politiques liées aux changements climatiques car ce sont elles qui activent les leviers permettant économie, efficacité et innovation en matière énergétique, via leurs politiques. Elles ont la responsabilité directe sur des investissements qui comptent parmi ceux qui ont la plus longue durée de vie : les bâtiments et les infrastructures de transport. Elles ont également en charge la répartition et l'organisation des activités sur le territoire à travers les décisions d'urbanisme et d'aménagement qu'elles prennent. Or, ce sont des décisions structurantes et peu réversibles.

\subsection{Le rôle des collectivités et intercommunalités}

10 La plupart des guides et rapports traitant de l'énergie au niveau local (MIES, 2007; Énergie-Cités, 2009 ; ADEME, 2009) désignent par le terme « collectivités » à la fois les collectivités territoriales (Conseils régionaux, Conseils généraux, communes) et intercommunalités (communautés urbaines, communautés d'agglomération, communautés de communes, syndicats mixtes), ainsi que les territoires de projets (Pays, parcs naturels régionaux). Afin d'éviter toute confusion, nous emploierons les expressions «collectivités et leurs groupements» ou «collectivités et intercommunalités » pour désigner l'ensemble de ces territoires.

11 Rappelons tout d'abord qu'en tant qu'acteurs de la politique locale, les collectivités françaises et leurs groupements contribuent directement pour environ $12 \%$ des émissions nationales de GES, « résultant de la gestion de leur patrimoine (flotte de véhicules, bâtiments publics) et des compétences qu'elles exercent (transport, déchets, chauffage urbain) (ADEME et al., 2005 : 5). Mais à travers leurs politiques d'aménagement du territoire et d'urbanisme, 
d'habitat, de transport, d'approvisionnement énergétique, elles agissent indirectement sur plus de $50 \%$ des émissions.

De manière générale, on s'accorde à distinguer cinq responsabilités des collectivités et de leurs groupements en matière d'énergie (Magnin, 1995 ; Tessier, 2005 ; AMORCE, 2008). Elles sont à la fois :

1. productrices d'énergie renouvelable ;

2. autorités concédantes du service public de distribution d'énergie ;

3. consommatrices se devant d'être exemplaires ;

4. aménageuses de l'espace urbain et du territoire ;

5. incitatrices de la sensibilisation et de l'éducation à l'environnement.

Elles ont donc un rôle majeur à jouer dans la mobilisation des acteurs locaux et la coordination des actions, à la fois pour le développement des énergies renouvelables et pour la maîtrise de la consommation énergétique. A. Smith rappelle que «les politiques visant à transformer les systèmes énergétiques existants en des systèmes qui privilégient les énergies renouvelables requièrent les efforts coordonnés d'une grande variété d'acteurs et d'institutions pour faire évoluer nos modes production $»^{4}$ (Smith, 2007 : 6268).

Par ailleurs, B. Laponche insiste sur le fait que «la stratégie de maitrise des consommations d'énergie est transversale à toutes les activités humaines » (2008: 13). Ainsi, dans une optique de réduction des émissions de GES, les collectivités et leurs groupements doivent à la fois mener des actions directement sur la chaîne énergétique (production-distributionconsommation), mais bien au-delà sur le triptyque espace-environnement-société. Cette approche locale est d'autant plus indispensable que les aménités et contraintes naturelles, économiques, démographiques et sociales du territoire, vont conditionner à la fois le versant ressources-production et le versant consommation-usages.

La nécessité de l'implication des échelons locaux pour atteindre des objectifs de développement durable, et plus particulièrement de réduction des émissions de GES, apparait dans les textes internationaux dès 1992 avec le Plan d'action pour le XXI ${ }^{e}$ siècle, défini lors du Sommet de la Terre de Rio de Janeiro. En France, le Programme national de lutte contre le changement climatique adopté en 2000 précise que «l'ancrage territorial constitue l'une des conditions du succès de la politique nationale de lutte contre l'effet de serre ». Cette affirmation est reprise dans le Plan climat national de 2004 qui met l'accent sur « la nécessaire prise en compte de la dimension territoriale dans la diminution des émissions nationales de GES».

Plus récemment, la loi de programmation relative à la mise en œuvre du Grenelle de l'environnement, dite loi Grenelle 1, stipule dans son article 2 que « les mesures nationales de lutte contre le changement climatique porteront en priorité sur la baisse de la consommation d'énergie des bâtiments et la réduction des émissions de gaz à effet de serre des secteurs des transports et de l'énergie ». Il est ainsi intéressant de constater que dans le Titre $\mathrm{I}^{\mathrm{er}}$ consacré à la Lutte contre le changement climatique, les thématiques traitées sont, dans l'ordre d'apparition des chapitres : le bâtiment, l'urbanisme, les transports, l'énergie et enfin la recherche dans le domaine du développement durable. Par ailleurs, la loi Grenelle 1 modifie le code de l'urbanisme en y introduisant les notions de réduction des émissions de gaz à effet de serre, de réduction des consommations d'énergie et d'économie des ressources fossiles (article 8). Cependant, la loi Grenelle 1 ne propose que les grandes orientations énergie-climat devant être intégrées dans les politiques publiques sans fixer de cadre réglementaire et contraignant. Celui-ci est précisé dans la loi Grenelle 2 (loi 
portant engagement national pour l'environnement, promulguée le 12 juillet 2010) qui « décline de manière concrète les orientations du "Grenelle $1 " \|^{5}$ et qui se présente comme "un énorme catalogue d'objectifs et de mesures annoncées "mais "dont la mise en œuvre effective dépend de décrets encore à prendre $»^{6}$.

De manière générale, les collectivités locales ne possèdent pas encore, ou pas suffisamment, cette culture énergie-climat et "éprouvent des difficultés à aborder les questions énergétiques, longtemps restées de la seule responsabilité de l'État" (Métreau, Laponche, 2003:5).

\section{3. L'exemplarité, les politiques publiques, la sensibilisation : les trois niveaux d'intervention des collectivités}

Les documents référents pour la construction d'une politique énergie-climat à l'échelle locale (ADEME et al., 2005 ; AMORCE, 2008 ; RAC-F, 2008 ; ADEME, 2009) distinguent trois niveaux d'intervention pour les collectivités et leurs groupements :

1. L'échelle interne ou «Actions sur le patrimoine et les services » : il s'agit des consommations de la collectivité (en énergie, en matériaux et en produits divers) pour offrir des services à la population (éducation, sport, culture, santé, loisirs, administration) ainsi que la conception des nouveaux bâtiments et équipements qui seront à leur tour consommateurs. Les collectivités et leurs groupements, à travers l'exemplarité de leurs modes de fonctionnement, doivent tracer la voie ;

2. L'échelle politiques publiques ou «Actions sur les politiques publiques »: il s'agit de tenir compte de la thématique énergie-climat dans l'ensemble des politiques publiques: urbanisme, mobilité et déplacements, conception de nouveaux quartiers et réhabilitation des anciens, approvisionnement énergétique local, politique globale des déchets, de l'eau et de l'assainissement...;

3. L'échelle externe ou "Actions sur les sphères d'influence »: il s'agit d'inciter, fédérer et mobiliser l'ensemble des acteurs du territoire (ménages, associations, entreprises et administrations) par des actions de sensibilisation et des mesures facilitatrices.

Les collectivités et leurs groupements doivent donc se fixer des objectifs clairs puis mettre en œuvre un certain nombre d'actions pour les atteindre, tant au niveau des consommations d'énergie que des émissions des GES, dans tous les secteurs d'activité et pour tous les niveaux d'intervention. Cette approche globale et transversale nécessite l'intégration de la thématique énergie-climat dans l'ensemble des politiques de la collectivité et devra conduire au niveau administratif à une implication de tous les services. Pour atteindre ces objectifs, les collectivités et leurs groupements peuvent s'appuyer sur les documents de planification et d'orientation et sur les dispositifs réglementaires déjà existants.

\section{Quels dispositifs pour intégrer la problématique énergie-climat dans les politiques énergétiques locales ?}

Dans la plupart des pays, dont la France, les collectivités locales et leurs groupements ne peuvent pas intervenir directement sur le marché de l'énergie (Bulkeley et al., 2009 : 15). Elles ne peuvent en effet mobiliser que partiellement les instruments économiques et 
réglementaires traditionnellement définis par les économistes. Cependant, l'évolution des politiques environnementales, et en particulier des politiques énergétiques et climatiques, qui nécessitent une approche transversale à l'ensemble des politiques publiques, fait émerger un certain nombre d'instruments «difficilement classables dans ce système binaire de classification " (Glachant, 2004,: 5; Mez, 2009,: 152). Ce sont ces instruments transversaux locaux peu étudiés dans la littérature que nous présentons ici.

\subsection{Des cadres d'action pour impliquer les acteurs du territoire}

21 Suite au Grenelle de l'environnement et dans la continuité du Plan Climat National de 2004, les collectivités territoriales de plus de 50000 habitants vont bientôt être dans l'obligation de réaliser un Plan Climat Énergie Territorial (PCET) tandis que les autres collectivités, intercommunalités et territoires de projet y seront fortement incitées. Actuellement, le PCET est une démarche volontaire. En juin 2010, on recensait 200 PCET engagés en France dans des collectivités territoriales, des intercommunalités et des territoires de projet (ADEME, 2010a). L' Agence de l'environnement et de la maîtrise de l'énergie (ADEME), sur son site consacré à l'observation des PCET, en donne la définition suivante : «le PCET est un projet territorial de développement durable dont la finalité première est la lutte contre le changement climatique. Institué par le Plan Climat National et repris par la loi Grenelle 1 et le projet de loi Grenelle 2, il constitue un cadre d'engagement pour le territoire. Il vise deux objectifs: (1) l'atténuation, il s'agit de limiter l'impact du territoire sur le climat en réduisant les émissions de gaz à effet de serre (GES) dans la perspective du facteur 4 (diviser par 4 les émissions d'ici 2050) ; (2) l'adaptation, il s'agit de réduire la vulnérabilité du territoire puisqu'il est désormais établi que les impacts du changement climatique ne pourront plus être intégralement évités. Un PCET se caractérise également par des ambitions chiffrées de réduction des émissions de GES et d'adaptation du territoire, dans des contraintes de temps »(ADEME, 2010b).

Le PCET doit répondre aux trois piliers du développement durable : la préservation de l'environnement, à travers les actions de réduction des émissions de GES; la justice sociale, avec la problématique de la précarité énergétique; et le progrès économique, avec l'emploi local généré grâce au développement d'une filière énergie renouvelable ou de maîtrise de la consommation. Il doit être conçu comme un cadre posé par la collectivité dans lequel s'inscrira l'ensemble des actions énergie-climat qu'elle mènera sur son territoire. C'est un outil permettant d'impliquer toutes les politiques publiques, tous les secteurs d'activité et tous les acteurs du territoire dans une démarche énergieclimat. Or, il existe déjà de nombreux dispositifs cadres qui proposent, dans un document de référence, une série de thèmes sur lesquels s'appuiera la collectivité pour mettre en place une politique énergétique ou plus globalement environnementale : agenda 21 local, Contrat d'objectif territorial (anciennement Aténée), démarche Cit'ergie. Ces dispositifs ne sont pas obligatoires; ils reposent sur la base du volontariat et tous les thèmes proposés dans le document de base ne doivent pas obligatoirement être traités.

Dans son guide Construire et mettre en ceuvre un Plan Climat Territorial publié en 2009, l'ADEME affirme que le PCET est une démarche complémentaire à l'Agenda 21 dont il peut constituer le volet « Énergie et lutte contre le changement climatique ». Afin de vérifier la complémentarité entre ces deux documents d'orientation et pour savoir s'ils pouvaient être mobilisés tous deux par les collectivités et leurs groupements pour mener une politique énergétique et environnementale cohérente sur les territoires, nous avons 
comparé les thèmes proposés dans les agendas 21, d'une part, et dans les PCET, d'autre part.

Les trois documents de référence pour la construction de PCET que sont le site Internet de l'ADEME Centre de ressources pour les Plans Climat-Energie Territoriaux, le guide de l'ADEME Construire et mettre en cuvre un Plan Climat Territorial (2009) et le kit méthodologique du Réseau Action Climat France (RAC-F) Comment réduire les émissions de gaz à effet de serre au niveau local et adapter les territoires aux effets des changements climatiques (2008), permettent dedéfinir 8 thèmes principaux pour les PCET :

1. production et distribution d'énergie ;

2. bâtiment;

3. urbanisme et aménagement;

4. transport;

5. agriculture, gestion des forêts et des espaces verts ;

6. mode de production et de consommation durable-gestion des déchets ;

7. biodiversité ;

8. économie locale.

Ces huit thématiques principales ne sont pas exhaustives et ne sont pas données ici par ordre de priorité. Elles sont totalement compatibles et recouvrent la moitié des six axes de l'agenda 21 national qui ont été définis par le Ministère de l'Écologie et du Développement Durable : la lutte contre le changement climatique ; le soutien aux filières environnementales et à l'économie sociale; l'éducation au développement durable; la préservation de la biodiversité ; la coopération internationale ; le respect de la diversité culturelle et la lutte contre les exclusions. Le schéma suivant nous permet d'appréhender le caractère transversal et complémentaire de ces deux démarches. 


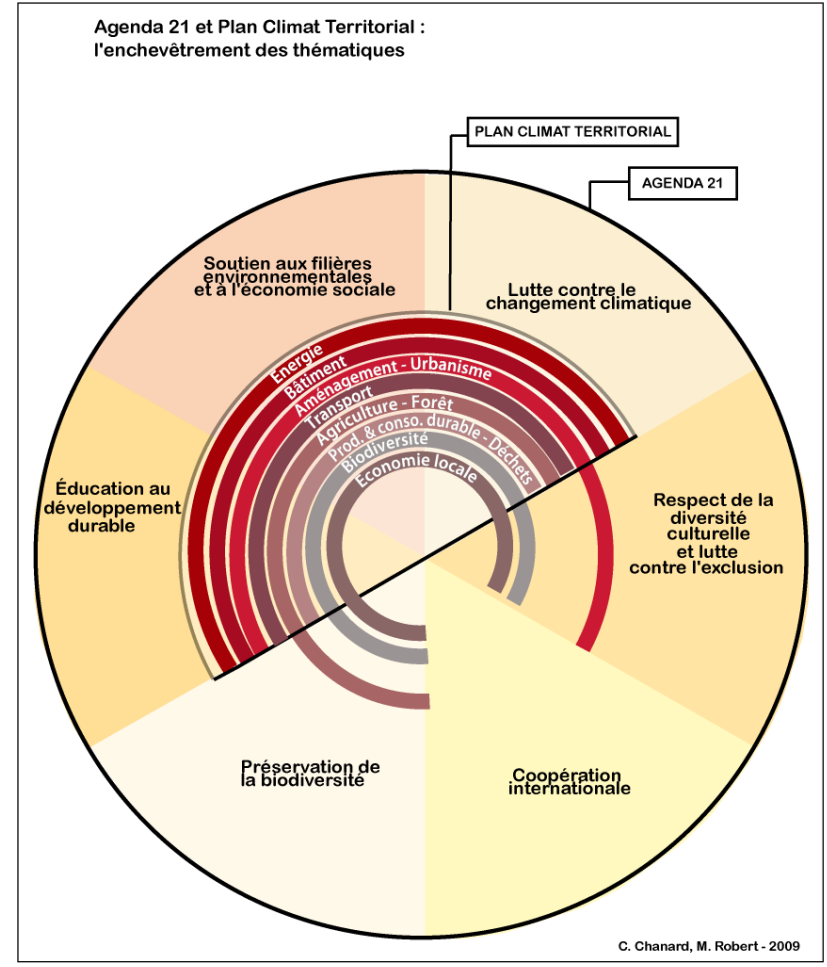

Nous voyons à travers ce schéma, qui synthétise les thématiques abordées dans un PCET et dans un agenda 21, que la frontière entre ces deux dispositifs n'est pas nette. Ainsi, bien que tous les organismes présentant ces dispositifs (ADEME, RAC, Énergie-Cités...) préconisent leur mise en place conjointe et complémentaire (que nous estimons également indispensable pour une politique énergétique cohérente et efficace), celle-ci n'apparaît pas clairement à première vue. La thématique " coopération internationale " par exemple, que nous n'avons pas incluse dans le Plan Climat Territorial, est intégrée par le RAC, et est évidemment indispensable à un développement soutenable et responsable du territoire. Autre exemple, nous avons considéré que les thématiques « agriculture et forêt » et « biodiversité » allaient au-delà du seul Plan Climat-Energie car elles intégraient directement des objectifs de préservation de la biodiversité et de coopération internationale.

Par ailleurs, rappelons que ces deux démarches sont, pour l'instant, volontaires et que les collectivités et leurs groupements peuvent engager des actions énergie-climat en dehors de ces cadres formels.

\subsection{Documents de planification et dispositifs réglementaires}

Le PCET constitue le principal outil transversal pour conduire une politiques énergieclimat sur un territoire, mais d'autres démarches plus thématiques peuvent aussi être engagées. L'ensemble de ces instruments transversaux, obligatoires ou volontaires, sont présentés dans le schéma 2. Les dispositifs de planification, qui permettent de fixer les grandes orientations de la politique énergétique sont distingués des outils d'accompagnement et de mise en œuvre, qui autorisent la mise en place d'actions concrètes sur le territoire. Cette liste n'inclut pas les outils de management 
environnemental, type norme ISO 14001 ou SD 21000, ceux-ci étant essentiellement à destination des entreprises ni les programmes européens et programmes et contractualisations spécifiques à certaines Régions afin de ne pas trop brouiller la lisibilité de l'ensemble.

Une classification de ces dispositifs a été réalisée selon plusieurs critères. Tout d'abord, les dispositifs ont été classés par grands secteurs d'intervention. Nous pouvons constater qu'ils correspondent globalement aux thématiques identifiées précédemment pour les PCET. Notons toutefois que les thématiques les moins touchées directement par les politiques énergie-climat, économie locale et production et consommation durable, ne disposent pas d'instruments spécifiques pour répondre à ces enjeux. Par ailleurs, aucun instrument lié directement à la thématique «biodiversité » n'a été identifié. Ceci illustre la pauvreté des possibilités d'action dans le volet « adaptation » des PCET.

Cette première classification a ensuite été subdivisée en fonction du type de dispositifs : leur mise en place nécessite-t-elle une approche globale de la problématique, est-ce un outil d'aide à la décision ou plutôt un instrument de régulation économique ? Cinq types d'instruments se dégagent : label, engagement, diagnostic, approche globale et régulation économique.

Enfin, il faut distinguer les dispositifs réglementaires des démarches volontaires, certaines étant réellement structurantes et d'autres étant des outils d'accompagnement. Cette dernière classification est évolutive, certains dispositifs testés par des collectivités ou des intercommunalités de façon volontaire dans un premier temps devenant par la suite obligatoires au niveau national (cela va notamment devenir le cas des Plans Énergie Climat Territoriaux).

Schéma 2 : Outils et dispositifs à disposition des collectivités et de leurs groupements pour mener leur politique énergétique

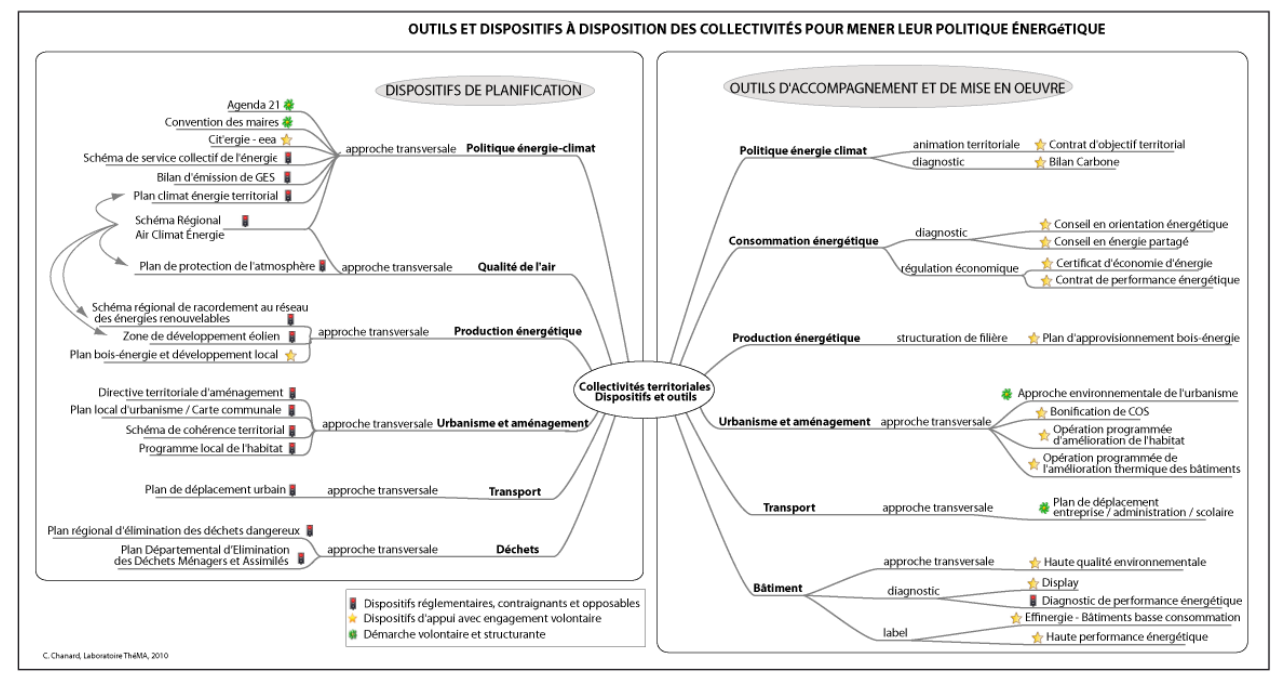

Un certain nombre de nouveaux dispositifs et outils de planification ont été introduit par la loi Grenelle 2. Tout d'abord, les PCET deviendront obligatoires pour toutes les collectivités de plus de 50000 habitants, tandis qu'un rapport sur la durabilité des équipements prévus devra être réalisé en amont des budgets. Les évolutions les plus importantes interviennent au niveau régional : obligation d'établir un bilan des émissions de GES, d'élaborer un Schéma régional de raccordement aux réseaux des énergies 
renouvelables et de proposer un Schémasrégional climat air énergie (SRCAE). Ces SRCAE devront être pris en compte dans tous les documents de planification aux échelons inférieurs et des liens de compatibilité seront imposés entre :

1. les Plans de protection de l'atmosphère (PPA, obligatoires pour toutes les agglomérations de plus de 250000 habitants);

2. les PCET à tous les niveaux d'échelle ;

3. les Schémas régionaux de raccordement aux réseaux des EnR ;

4. les zones de développement éolien.

Nous voyons apparaître, à travers ce schéma récapitulatif, la diversité des thématiques concernées, la multiplicité des dispositifs et instruments pouvant être mobilisés dans le cadre d'une politique énergie-climat et le niveau de contrainte qu'ils imposent. Cette multiplicité difficilement compréhensible, et qui entraîne parfois des superpositions dans les actions et les périmètres d'intervention, peut également s'avérer positive. Elle peut en effet permettre aux élus et techniciens locaux de s'adapter au mieux aux caractéristiques naturelles, sociales, économiques et organisationnelles de leur territoire de gestion, et faire apparaître de nouvelles formes de gestion, évoluant en fonction des nouveaux défis se présentant aux collectivités locales et à leurs groupements. Encore faut-il que les compétences entre collectivités soient clairement définies et que l'accès à l'information soit direct et de qualité.

\section{De nouveaux défis organisationnels pour une réponse efficace}

La complexité du système sur lequel agir (le système territorial) impose un profond changement de paradigme et une modification radicale des modes de gestion et de la répartition des compétences de chacun des acteurs concernés. Les collectivités et leurs groupements, dans ce contexte, restent des acteurs majeurs du changement ${ }^{7}$. Elles doivent fournir à la fois les éléments de diagnostic pour convaincre de la pertinence des actions ainsi que les leviers d'actions incitatifs ou contraignants à même d'influencer significativement l'ensemble du système. Pour atteindre ces objectifs, trois défis doivent être relevés: la connaissance des territoires et de leur système énergétique, le décloisonnement des actions et la clarification des compétences entre les collectivités et leurs groupements. Les récents débats et les propositions issues du Grenelle environnement témoignent de l'évolution dans la manière d'aborder le sujet, mais ne semblent pas encore suffisants pour un changement significatif.

\subsection{Connaître le fonctionnement énergétique des territoires pour poser des diagnostics précis}

Les exigences qu'imposent la situation énergétique et environnementale et l'urgence à y répondre appellent la réalisation de diagnostics précis. Il faut être capable d'identifier les potentiels d'économie d'énergie, les performances des territoires en matière de production, de consommation, les actions d'ores et déjà menées en les replaçant dans leur contexte et en analysant leurs performances, en identifiant des profils de comportement énergétique des territoires (Fléty et al., 2009). Ces attentes doivent passer par la mise à disposition d'indicateurs d'état, de réalisation, de performance permettant 
d'appréhender l'hétérogénéité et la variabilité spatiale des phénomènes sur le territoire (Joerin et al., 2005). Cette connaissance apparaît comme le prérequis indispensable à la mise en œuvre de politiques cohérentes et efficaces.

Cependant, il reste aujourd'hui très malaisé de poser des diagnostics énergétiques réellement adaptés à l'ampleur des défis. De nombreuses études sont pourtant produites (cadastre des émissions, études sur la mobilité des ménages, diagnostics de performance énergétique de bâtiments). Pourtant, il est bien difficile de mettre en musique l'ensemble de ces éléments épars dont l'analyse conjointe permettrait pourtant une qualification plus fine des comportements énergétiques territoriaux. Ainsi, l'efficacité institutionnelle est faible car l'accès à l'information est limité suite à la rétention d'informations détenues par des acteurs clés (entreprises de distribution d'énergie par exemple, mais aussi bailleurs sociaux ou entrepreneurs), la non-coordination des collectivités et de leurs groupements, la méconnaissance de l'existant, l'absence d'indicateurs synthétiques et d'outils de suivi performants. Cette situation est d'autant plus difficile à faire évoluer que la question de l'articulation et la complémentarité des niveaux observés et des indicateurs produits s'était déjà posée lorsque se sont multipliés les dispositifs d'observation, sans avoir été résolue (Noucher et al., 2008).

\subsection{Décloisonner les compétences, les acteurs et les thématiques}

Le principal défi auquel sont confrontées les collectivités locales et les intercommunalités pour répondre aux objectifs du facteur 4 est le décloisonnement: décloisonnement des compétences, décloisonnement des thématiques, décloisonnement des acteurs. Ainsi, F. Mancebo (2009: 18) identifie trois difficultés majeures dans la mise en place de politiques de développement durable à l'échelle locale que l'on retrouve lors de la construction de politiques énergie-climat ambitieuses et cohérentes.

Tout d'abord, il n'existe généralement pas d'articulation des compétences, des dispositifs, des ambitions et des politiques entre les différentes collectivités (régions, départements, communes) et intercommunalités et territoires de projet (pays, communautés urbaines, d'agglomération, de communes).

Ensuite, «beaucoup de collectivités s'avèrent incapables de sortir d'une organisation sectorielle incompatible avec la transversalité de l'action publique propre au développement durable ». Dans son étude sur le Plan climat de Paris, C. Ghorra-Gobin (2009) fait le même constat. En effet, la stratégie énergie-climat de la municipalité apparaît dans trois documents différents produits par trois services différents: le Plan local d'urbanisme, le Plan de déplacement de Paris et les Opérations programmées d'amélioration de l'habitat.

Enfin, la participation des acteurs du territoire, et en particulier des habitants, est difficile à mettre en place et à maintenir dans le long terme. Nous fonctionnons en effet depuis des décennies dans une culture du découpage: découpage sectoriel entre de grands domaines (transport, habitat...), découpage spatial et emboîtement des territoires de gestion dont les modes de fonctionnement ne sont pas vraiment clairs. Tout cela aboutit à une atomisation des compétences et à une absence de gouvernance ainsi qu'à un empilement législatif de moins en moins maitrisable. En toute logique, il devient donc difficile de mettre en œuvre des approches transversales de la problématique énergétique. 


\subsection{Clarifier et rendre efficace la répartition des compétences entre collectivités}

41 Nous avons démontré dans cet article la nécessité d'une approche transversale de l'énergie, tant du point de vue des thématiques à intégrer que des acteurs à mobiliser. Le recensement de l'ensemble des dispositifs et outils à disposition des collectivités locales et de leurs groupements nous permet d'apercevoir en filigrane les actions concrètes pouvant être réalisées sur le territoire, leur imbrication et interdépendance. Cependant, nous n'avons pas encore évoqué le problème de la répartition des compétences entre les collectivités locales et leurs groupements. En particulier, nous n'avons pas fait apparaître sur le schéma précédent le nom des collectivités pouvant mobiliser les dispositifs. Plusieurs raisons à cela: tout d'abord, certains dispositifs, en particulier les cadres d'actions (agenda 21, PCET ou Cit'ergie), peuvent être mis en place à différents niveaux (communes, agglomération et Région par exemple); ensuite parce que suivant les cas, certaines compétences ont été transférées de la commune à l'intercommunalité et que l'insertion de cette information complexifierait grandement la lecture du schéma. Enfin, nous souhaitions mettre en évidence l'ensemble des thématiques devant tenir compte de la problématique énergétique sur un territoire, sans tenir compte des découpages administratifs.

42 Toutefois, il est essentiel de connaître le fonctionnement institutionnel, la répartition des compétences entre les collectivités et intercommunalités et la compatibilité entre les documents de planification pour proposer la mise en œuvre d'actions concrètes. En effet, chaque collectivité ne pourra agir que sur son territoire d'action en fonction des compétences qui lui sont attribuées.

Si l'on se penche sur la thématique transversale "aménagement et urbanisme », nous constatons que les communes et les intercommunalités sont concernées, mais que leur implication peut varier en fonction du transfert de compétence. Le Plan local d'urbanisme (PLU) - ou la carte communale pour les petites communes - qui définit et règle l'usage des sols sur l'ensemble du territoire concerné, est élaboré au niveau de la commune ou de l'intercommunalité. La Bonification de coefficient d'occupation du sol est en revanche gérée par le conseil municipal; il permet aux communes d'autoriser à construire $20 \%$ de surface supplémentaire par rapport au PLU si les constructions ont une performance supérieure à la consommation conventionnelle de référence. Le même outil s'applique si le projet inclut une solution faisant appel aux énergies renouvelables. Le Programme local de l'habitat (PLH) est lui exclusivement géré par l'intercommunalité, tandis que les opérations programmées d'amélioration de l'habitat et d'amélioration thermique des bâtiments sont instaurées par la commune ou l'intercommunalité selon les cas. Enfin, le Schéma de cohérence territorial (SCOT), qui fixe les orientations fondamentales de l'organisation du territoire et de l'évolution des zones urbaines, est réalisé à l'échelle de plusieurs communes ou groupements de communes.

L'exemple que nous venons de développer, axé sur les documents d'urbanisme les plus importants, nous montre déjà la complexité du système d'acteurs et l'emboîtement des compétences de chacun dont la traduction apparaît dans les nombreux documents (PLU, SCOT, PLH, etc.). Cette situation est d'autant plus inquiétante que les problématiques énergétiques et climatiques ne sont pas, pour l'instant, au centre des préoccupations 
d'aménagement du territoire, rendant bien souvent caducs les efforts réalisés en matière de réduction des consommations énergétiques et d'émissions des GES.

Par ailleurs, une étude réalisée au niveau européen sur l'évaluation des agendas 21 a démontré que, dans la majorité des cas, "cette démarche reste souvent trop proche des services en charge de l'environnement, et tend à promouvoir des politiques finalement sectorielles, environnementales " (ICLEI/DIFU, 1999, cité in Emilianoff, 2005: 2). On peut donc légitimement s'interroger sur la place que prendront les PCET dans l'organisation administrative des collectivités et intercommunalités.

Enfin, les outils de cadrage et de planification tels que les agendas 21 et les PCET ont pour objectif de faire évoluer les comportements des acteurs du territoire en affichant clairement des objectifs de réduction des consommations d'énergie et d'émissions de GES. Ce ne sont toutefois pas des démarches obligatoires (jusqu'à présent) et elles laissent une grande latitude dans les objectifs, la méthodologie et l'évaluation des actions. Cela pose donc la question de leur suivi dans la durée et de leur évaluation. Ne passe-t-on pas d'un outil d'affichage à un autre, sans réaliser de véritable évaluation des actions réalisées dans ces cadres?

\section{Conclusion}

Cet article présente l'ensemble des dispositifs réglementaires et volontaires sur lesquels peuvent s'appuyer les collectivités et leurs groupements afin de réduire les consommations énergétiques et les émissions des GES sur leur territoire. La mobilisation de ces outils doit leur permettre d'intégrer la thématique énergie-climat dans l'ensemble des politiques publiques, d'aménager le territoire, de repenser l'espace et son appropriation.

Cependant, la plupart de ces outils, qui sont généralement des outils thématiques développés pour un domaine d'action particulier, ne sont pas toujours identifiés comme des leviers pouvant être actionnés pour atteindre les objectifs énergétiques et climatiques. Ainsi, s'il semble inutile d'ajouter de nouveaux documents de planification à la multitude de ceux déjà existants, des efforts doivent encore être réalisés pour que les collectivités et intercommunalités les connaissent et les mobilisent dans cette optique. Chaque collectivité, intercommunalité et territoire de projet doit à présent identifier dans l'ensemble des dispositifs de planification et outils d'accompagnement déjà mis en œuvre sur son territoire dans différents domaines, ceux pouvant être mobilisés pour construire une politique énergétique et climatique ambitieuse.

Une autre difficulté pour la construction de politiques énergétiques locales cohérentes entre les différents niveaux de collectivités et groupements est la question de l'emboîtement des compétences. La complexité de nos institutions et l'empilement des niveaux de décision favorisent une méconnaissance globale du rôle des différents acteurs. Les PCET tels qu'ils sont définis dans les lois Grenelle doivent apporter une coordination des actions aux échelons locaux. Cependant, il est actuellement prévu que toutes les collectivités de plus de 50000 habitants devront posséder leur PCET, sans que la question de la compatibilité ne soit posée. Ainsi, à la suite du Grenelle environnement, une large réflexion doit à présent être lancée afin de clarifier les compétences de chaque territoire de gestion. 
De gros efforts devront être fournis par les collectivités et leurs groupements afin de changer les modes de fonctionnement, d'adapter l'organisation des acteurs locaux de l'énergie et les organigrammes administratifs et d'assurer la transparence informationnelle autour de cette thématique.

\section{BIBLIOGRAPHIE}

ADEME, Ministère de l'écologie et du Développement Durable, Association des Maires de France, 2005, Un plan climat à l'échelle de mon territoire, [ND]

ADEME, 2009, Construire et mettre en œuvre un Plan Climat Territorial, Guide méthodologique, [ND]

ADEME, 2010a, Centre de ressources pour les Plans Climat-Énergie Territoriaux, [en ligne], consulté en juin 2010, disponible sur : http://www.pcet-ademe.fr/

ADEME, 2010b, « Élus : les réponses à vos questions - Qu'est-ce qu'un Plan Climat-Energie Territorial », Centre de ressources pour les Plans Climat-Energie Territoriaux de l'ADEME, [en ligne], consulté le 10 juin 2010, disponible sur http://www.pcet-ademe.fr/elus/qu\%E2\%80\%99est-qu\% E2\%80\%99un-plan-climat-energie-territorial\%C2\%A0

AMORCE, 2008, L'élu, l'énergie et le climat, [ND]

Bulkeley H., Schroeder H., Janda K., Zhao J., Armstrong A., Chu S. Y., Ghosh S., 2009, "Cities and Climate Change: the role of institutions, governance and urban planning, Fifth Urban Research Symposium 2009: cities and Climate Change: responding to an Urgent Agenda, 28-30 June 2009, Marseille-France

CITEPA (Centre interprofessionnel technique d'étude de la pollution atmosphérique), 2010, Inventaire des émissions de gaz à effet de serre en France au titre de la Convention cadre des Nations-Unies sur les changements climatiques

Emelianoff C., «Les agendas 21 locaux : quels apports sous quelles latitudes? », Développement durable et territoires, [en ligne], Dossier $4:$ La ville et l'enjeu du Développement Durable, mis en ligne le 31 mai 2005, consulté en novembre 2009, URL : http://developpementdurable.revues.org/ index532.html

Énergie-Cités, 2009, Les Plans Climat Énergie Territoriaux, Guide, [en ligne], consulté en novembre 2009, URL : http://www.energie-cites.eu/Qu-est-ce-qu-un-Plan-climat

Fléty Y., Antoni J.-P. , Vuidel G., Sède-Marceau (de) M.-H., 2009; Towards local energy performance indicators: territorial energy labeling, 16th European Colloquium on Theoretical and Quantitative Geography (ECQTG09), 4th-8th September, 2009, Maynooth, Ireland

Ghorra-Gobin C., 2009, "Climate change and public policies at the local scale: The complexity of the task ", Fifth Urban Research Symposium 2009: Cities and Climate Change: Responding to an Urgent Agenda, 28-30 June 2009, Marseille-France

Glachant M., 2004, Les instruments de la politique environnementale, Polycopié du cours de Microéconomie de l'environnement II, DEA Économie de l'Environnement et des Ressources Naturelles 
IEA (International Energy Agence), 2009, Key World Energy Statistics, [en ligne], consulté en novembre 2009, URL : http://www.iea.org/Textbase/publications/free_new_Desc.asp? PUBS_ID $=1199$

Jancovici J.-M., 2004, «L'homme et l'énergie : des amants terribles », La Jaune et la Rouge, sept. 2004, [en ligne], consulté en décembre 2007

Joerin F., Nembrini A., Billeau S., Desthieux G.,. 2005, « Indicateurs spatialisés : un instrument de participation en aménagement du territoire ", Revue internationale de géomatique, 15(1), p. 33-61

Laponche B., 2008, Prospective et enjeux énergétiques mondiaux - Un nouveau paradigme, AFD Document de travail $n^{\circ} 59$

Magnin G., 1995, «Ville et énergie faut-il redéfinir la place des collectivités territoriales dans les politiques énergétiques? ", Revue de l'énergie, n473, p. 806-813.

Mancebo F., 2009, « Des développements durables. Quel référentiel pour les politiques de développement durable en Europe ? ", Cybergeo: European Journal of Geography, [en ligne], Espace, Société, Territoire, document 438, mis en ligne le 10 février 2009, consulté en novembre 2009, URL : http://www.cybergeo.eu/index21987.html

Métreau E., Laponche B., 2003, Étude de faisabilité d'une initiative d'animation des réseaux d'acteurs français impliqués dans les actions énergétiques territoriales, Volume 1 : Rapport, ICE, Etude réalisée pour l'ADEME

Mez L., 2009, Zukünftiger Ausbau erneuerbare Energieträger unter besonderer Berücksichtigung der Bundesländer, Otto-Suhr-Institut für Politikwisschenschaft, Université Libre de Berlin, Rapport réalisé pour le Ministère fédéral de l'environnement, de la protection de la nature et de la sécurité nucléaire

MIES (Mission Interministérielle de l'Effet de Serre), 2007, Plans Climat Territoriaux : des territoires en action, 21 collectivités engagées dans la relève du défi climatique, $1^{\text {er }}$ retour d'expérience 2007, Paris : MEDAD

Noucher M., Golay F., Sède-Marceau (de) M-H., Pornon H., 2008, Pas de décision collective sans appropriation individuelle : Enjeux et limites des technologies de l'information géographique au service du " décider ensemble ", Communication présentée à la conférence OPDE 2008, Québec, 5 et 6 juin

RAC-F (Réseau Action Climat France), 2008, Comment réduire les émissions de gaz à effet de serre au niveau local et adapter les territoires aux effets des changements climatiques, Kit d'information sur les Plans Climat-Énergies Territoriaux

Smith A., 2007, "Emerging between: The multi-level governance of renewable energy in the English regions", Energy Policy, 35 (2007), p. 6266-6280

Stern N., 2006, Stern Review on the Economics of Climate Change, HM Treasury and Cabinet

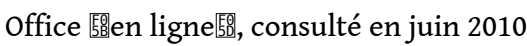

Tessier P., 2005, «Énergie et territoire, quoi de neuf en attendant le $1^{\mathrm{er}}$ juillet 2007 ? » Techni.Cités , n97, p. 26-33

Watson, R.T. , Zinyowera M.C., Moss R.H., 1996, Techniques, politiques et mesures d'atténuation des changements climatiques, Document technique I du GIEC, [en ligne], consulté en novembre 2009, URL : http://www.ipcc.ch/pdf/technical-papers/paper-I-fr.pdf

Loi $n^{\circ} 2005-781$ du 13 juillet 2005 de programme fixant les orientations de la politique énergétique Loi n²009-967 du 3 août 2009 de programmation relative à la mise en œuvre du Grenelle de l'environnement, dite Loi Grenelle I 
Loi $\mathrm{n}^{\circ} 2010-788 \mathrm{du} 12$ juillet 2010 portant engagement national pour l'environnement

\section{NOTES}

1. Nous tenons à remercier l'équipe de la direction régionale de l'ADEME Franche-Comté, qui a encadré le stage de $\mathrm{M}$. Robert et qui a apporté de nombreuses remarques et suggestions sur notre travail. Nous remercions également les rapporteurs d'évaluation rendus anonymes qui ont relu notre proposition. Leurs corrections et commentaires nous ont permis de préciser notre propos.

2. Notons que la contribution de chaque État membre à l'effort communautaire pour atteindre cet objectif a été fixé à partir des émissions de l'année 2005 䈏décision nº66/2009/CE du Parlement européen et du Conseil du 23 avril 2009, article 3 et annexe II 閲.

3. Cela se traduit en particulier par l'apparition de nouveaux concepts comme les Négawatts ou le cinquième combustible, considérés par certains comme des filières de production (d'économie d'énergie) à part entière.

4. "Policy objectives for transforming existing energy systems into ones with greater renewable energy content require co-ordinated efforts and changes among many different actors, institutions and artefactsPolicy objectives for transforming existing energy systems into ones with greater renewable energy content, require co-ordination efforts and changes among many different actors, institutions and artefacts" ; Traduction des auteurs.

5. Portail du Gouvernement, en ligne, consulté en novembre 2010, disponible sur http:// www.gouvernement.fr/gouvernement/projet-de-loi-grenelle-2-le-temps-de-l-application-et-dela-territorialisation

6. Vie Publique, Environnement: trois ans après, vers la dernière étape du Grenelle, publié le 21.07.2010, disponible sur : http://www.vie-publique.fr/actualite/dossier/environnementgrenelle/environnement-trois-ans-apres-vers-derniere-etape-du-grenelle.html?xtor=EPR-56

7. Sur cette thématique, voir les travaux de S. A Hammer sur les moyens d'action des autorités locales dans le domaine énergie-climat et notamment son article présenté en 2009 lors du 5th Urban Research Symposium 2009: Cities and Climate Change: Responding to an Urgent Agenda intitulé Capacity to Act: The critical determinant of local energy planning and program implementation.

\section{RÉSUMÉS}

Les collectivités ont traditionnellement des responsabilités multiples en lien avec l'énergie et peuvent, par conséquent, fortement influer sur la réduction des émissions de gaz à effet de serre. Elles agissent directement sur la chaîne énergétique (production-distribution-consommation) à travers leur patrimoine, mais aussi sur le triptyque espace-environnement-société, en particulier à travers leurs choix d'aménagement. Cet article réalise un tour d'horizon des outils de régulation et dispositifs de planification pouvant être mobilisés par les collectivités locales pour construire des politiques énergétiques et climatiques qui intègrent l'ensemble des politiques publiques et des actions réalisées sur leur territoire.

Local authorities have, traditionally, many responsibilities in the energy field and, therefore, can influence on greenhouse gas emissions. They directly impact on energy chain with consumption in public buildings, supply networks and energy production equipments. They also impact on the 
triptych space-society-environment with land planning choices. The paper realises an overview of policy instruments and planning instruments that could be used by French local communities in order to structure energy and climate policy, taking into account whole public policies and actions realised on the area.

\section{INDEX}

Keywords : energy policy, land planning, local communities, planning instruments

Mots-clés : aménagement, collectivités locales, dispositifs de planification, politique énergétique

\section{AUTEURS}

\section{CAMILLE CHANARD}

Camille Chanard est doctorante en géographie au laboratoire ThéMA (UMR 6049 du CNRS et des Universités de Bourgogne et de Franche-Comté) bénéficiant d'une bourse ADEME. Ses travaux portent sur l'organisation des acteurs et l'intégration des caractéristiques du territoire dans les politiques énergétiques locales. Laboratoire ThéMA (Théoriser et Modéliser pour Aménager) UMR 6049 CNRS - Université de Franche-Comté 32, rue Mégevand - 25030 Besançon Cedex France Tél. (33) (0)3 81665172 camille.chanard@univ-fcomte.fr

\section{MARIE-HÉLÈNE DE SÈDE-MARCEAU}

Marie-Hélène de Sède-Marceau est professeur de géographie au laboratoire ThéMA (UMR 6049 du CNRS et des Universités de Bourgogne et de Franche-Comté). Elle enseigne et mène des recherches dans le domaine des sciences et technologies de l'information géographique appliquées notamment à la gestion et à la planification des ressources et de l'environnement. Laboratoire ThéMA (Théoriser et Modéliser pour Aménager) UMR 6049 CNRS - Université de Franche-Comté 32, rue Mégevand - F-25030 Besançon cedex Tél. (33) (0)3 81665497 mariehelene.de-sede-marceau@univ-fcomte.fr

\section{MICAEL ROBERT}

Micael Robert est titulaire d'un master en Gestion durable de l'environnement et des territoires effectué à l'Université de Franche-Comté. Il a effectué son stage de fin d'étude à la direction régionale de l'ADEME Franche-Comté où il était en charge de réaliser un référentiel Plan Climat Territorial à l'échelle d'un territoire de projet. Il est actuellement Conseiller en Énergie Partagé au sein du Syndicat Mixte du Pays Doubs Tél. (33) (0)3 81844248 mrobert.doubs.central@orange.fr 